\title{
SUBGROUPS OF FINITELY PRESENTED SOLVABLE LINEAR GROUPS
}

BY

\author{
MICHAEL W. THOMSON
}

\begin{abstract}
Let $G$ be a finitely generated solvable linear group. It is shown that there exists a finitely presented solvable linear group $H$ with $G$ embedded in $H$.
\end{abstract}

1.1. The complexity of the subgroup structure of finitely presented solvable groups has only recently begun to emerge (e.g. see the papers of Gilbert Baumslag [2]-[6]). Among the results is the following theorem of Baumslag [2]: Every finitely generated metabelian group can be embedded in a finitely presented metabelian group. This indicates that the subgroup structure of finitely presented solvable groups is suprisingly complex. In fact, in [3] Baumslag has demonstrated the existence of a finitely presented solvable group which is not residually finite.

Despite this knowledge many open problems remain, e.g.: Is a finitely presented solvable group hopfian? In this regard it is worth noting that Philip Hall's old question (see [11]) as to whether a finitely presented solvable group necessarily satisfies the maximal condition for normal subgroups is still openV. N. Remeslennikov's assertion in [14] is unfortunately incorrect because his group does satisfy the maximal condition for normal subgroups.

The object of this paper is to throw some additional light on the nature of finitely presented solvable groups by proving the following:

THEOREM 1. Let $R$ be a commutative associative ring with 1 and $n$ a positive integer. Further, let $G$ be a finitely generated multiplicative group of $n \times n$ triangular matrices over $R\left({ }^{1}\right)$. Then there exists a commutative associative ring $S$ with 1 and a finitely presented multiplicative group $H$ of $n \times n$ triangular matrices over $S$ with $G$ embeddable in $H$. Moreover if $R$ is also a domain, then $S$ can be taken to be the field of fractions of $R$.

(The analogue of this theorem for associative algebras has recently been obtained by Gilbert Baumslag [7].)

Received by the editors January 5, 1976.

AMS (MOS) subject classifications (1970). Primary 20F05, 20E15, $20 \mathrm{H} 25$.

Key words and phrases. Finitely presented, solvable, linear groups.

(1) By definition a matrix is triangular if all its elements above the main diagonal are zero.

- American Mathematical Society 1977 
Remeslennikov [15] has pointed out that if $G$ is any finitely generated metabelian group there exists a commutative associative ring $R$ with 1 such that $G$ is embedded in the multiplicative group of $2 \times 2$ triangular matrices over $R$. Hence for $n$ equal to 2, Theorem 1 reduces to Baumslag's Theorem [2].

1.2. Theorem 1 admits a slight, but pleasing, generalization for solvable linear groups:

THEOREM 2. Every finitely generated solvable linear group can be embedded in a finitely presented solvable linear group.

Here a linear group is simply a multiplicative group of nonsingular matrices with coefficients in some commutative field.

There are some other consequences of Theorem 1 that are worth observing. First of all we note that every countable free nilpotent group of any given class $c$ has a faithful representation as a linear group (e.g. seeB. A. F. Wehrfritz [17, p. 34]). It is not difficult to embed this linear representation into a finitely generated solvable linear group and thereby prove

COROLLARY 1. A free nilpotent group of any given class $c$ and countable rank can be embedded in a finitely presented solvable linear group.

Similarly using the appropriate matrix representations due to D. I. Ėidel'kind [8] in the first case and C. K. Gupta [9] in the second, one can also prove the further corollaries.

COROLlARY 2. A finitely generated free group in the product variety $\mathfrak{N}_{c} \mathfrak{A}$ can be embedded in a finitely presented linear group in the same variety (here $\mathfrak{N}_{c}$ is the variety of all nilpotent groups with class less than or equal to $c$ and $\mathfrak{A}$ is the variety of all abelian groups).

COROLLARY 3. The free center-by-metabelian group of rank 3 can be embedded in a finitely presented solvable linear group.

It should be noted that the result of D. I. Ėidel'kind [8] was also proved by N. S. Romanovskii [16] and C. K. Gupta and N. D. Gupta [10].

1.3. The arrangement of this paper is as follows. First we shall take care of the notation and all the other preliminaries in \$2. The proof of Theorem 1 begins in $\$ 3$ and is concluded in $\$ 7$. The proofs of Theorem 2 and Corollary 1 are contained in $\S \S 8$ and 9 respectively. Finally, in $\S 10$ we shall explain why Remeslennikov's assertion in [14] is incorrect.

2. In this section we record some of the notation and notions we shall need in the sequel.

As usual if $a$ and $b$ are elements of a group $G$ and $H$ is a subgroup of $G$ then we define 


$$
[a, b]=a^{-1} b^{-1} a b, \quad a^{b}=b^{-1} a b \quad \text { and } \quad H^{b}=b^{-1} H b .
$$

If $t$ is an element of $G$ and $f(x)=n_{0}+n_{1} x+\cdots+n_{d} x^{d}$ is an element of the polynomial ring $Z[x]$ then we shall write

$$
a^{f(t)}=a^{n_{0}}\left(a^{n_{1}}\right)^{t} \cdots\left(a^{n_{d}}\right)^{t^{d}} .
$$

We shall need two lemmas of Baumslag [2], which we record here as Lemma 1 and Lemma 2.

LEMMA 1. Let $R$ be a finitely generated commutative associative ring with 1 and let $s$ be a unit of $R$ with infinite order. Then there exists a nonzero divisor $f$ in $R$ of the form

$$
f=1+c_{1} s+\cdots+c_{d-1} s^{d-1}+s^{d}
$$

where $d, c_{1}, \ldots, c_{d-1}$ are integers and $d$ is positive.

LEMMA 2. Let $a, b, t, u$ be elements of a group and let $d$ be a positive integer. Suppose that $[t, u]=1$ and that $\left[a, b^{t^{n}}\right]=1$ whenever $-d \leqslant n \leqslant d$. In addition suppose that $a^{u}=a^{f(t)}, b^{u}=b^{f(t)}$ where $f(x)=1+c_{1} x+\cdots+c_{d-1} x^{d-1}$ $+x^{d}$ is an element of the polynomial ring $Z[x]$. Then $\left[a, b^{t^{i} u^{j}}\right]=1$ for all integers $i, j$.

Let $R$ be an associative ring with 1 and $n$ a positive integer. GL $(n, R)$ denotes the group of units of the ring of $n \times n$ matrices over $R$ with identity $1_{n}$. The set of triangular matrices in $\mathrm{GL}(n, R)$ forms the subgroup of $\mathrm{GL}(n, R)$ which is denoted by $\operatorname{Tr}(n, R)$. As usual if $A$ is an element of $\mathrm{GL}(n, R)$ we will write $A=\left(a_{i, j}\right)$ where $a_{i, j}$ is the entry appearing in the $i$ th row and $j$ th column of the matrix $A$.

Throughout the remainder of this paper a ring will be taken to mean a commutative associative ring with a 1 . The facts about these rings which are used in $\$ 4$ may be found in Atiyah and Macdonald [1].

3. Our ultimate objective is to show that if $R$ is a ring, $n$ a positive integer and $G$ a finitely generated subgroup of $\operatorname{Tr}(n, R)$ then there exist a ring $S$ and a finitely presented subgroup $H$ of $\operatorname{Tr}(n, S)$ with $G$ embeddable in $H$. Moreover if $R$ is also a domain, then $S$ can be taken to be the field of fractions of $R$.

The proof of these assertions is divided into four sections. The first section deals with constructing a suitable ring to play the role of $S$. Secondly, we examine a special subgroup $H$ of $\operatorname{Tr}(n, S)$ which contains an isomorphic copy of $G$. The final step of the proof is devoted to showing that $H$ is a finitely presented group. 
4. Let $R$ be a ring, $n$ a positive integer and $G$ a finitely generated subgroup of $\operatorname{Tr}(n, R)$. Suppose $G$ is generated by the matrices $g_{1}, \ldots, g_{m}$.

We can assume that $R$ is finitely generated. Thus, by the Lasker-Noether theorem, there exists a primary decomposition of the zero ideal, say $q_{1} \cap \cdots$ $\cap q_{p}=(0)$. Let $R_{i}$ be the quotient ring $R / q_{i}(1 \leqslant i \leqslant p)$. Then $R$ is embedded in the direct sum $R^{\prime}$ of the rings $R_{i}$. Since the zero divisors of $R_{i}$ are nilpotent elements, by localizing the nonzero divisors of $R_{i}$ if necessary, we can assume every element of $R_{i}$ is a unit or a nilpotent element $(1 \leqslant i \leqslant p)$. It follows that for our purposes we can assume the additive group of $R^{\prime}$ is generated by units of $R^{\prime}$. Therefore we can choose a finite set of units $s_{1}, \ldots, s_{q}$ of $R^{\prime}$ such that the elements of $R^{\prime}$ appearing below the main diagonal of the generating matrices $g_{1}, \ldots, g_{m}$ of $G$ are all contained in the additive subgroup of $R^{\prime}$ generated by $s_{1}, \ldots, s_{q}$. In addition let $s_{q+1}, \ldots, s_{r}$ be the units of $R^{\prime}$ which appear on the main diagonal of the generating matrices $g_{1}, \ldots, g_{m}$ of $G$.

Now let $R^{\prime \prime}$ be the subring with 1 of $R^{\prime}$ generated by the elements $s_{1}, \ldots, s_{q}, s_{q+1}, \ldots, s_{r}$ and their inverses. By relabeling if necessary, we can assume that $s_{1}, \ldots, s_{q}$ are of infinite order and that $s_{q+1}, \ldots, s_{r}$ are respectively of finite order $e_{q+1}, \ldots, e_{r}$. According to Lemma 1 for each $s_{i}$ of infinite order there exists a nonzero divisor $f_{i}$ in $R^{\prime \prime}$ of the form

$$
f_{i}=1+c_{i, 1} s_{i}+\cdots+c_{i, d_{i}-1} s_{i}^{d_{i}-1}+s_{i}^{d_{i}}
$$

where $d_{i}, c_{i, 1}, \ldots, c_{i, d_{i}-1}$ are integers and $d_{i}$ is positive $(1 \leqslant i \leqslant q)$. Therefore, by localizing $R^{\prime \prime}$ if necessary, we can assume $f_{i}$ is a unit in $R^{\prime \prime}(1 \leqslant i$ $\leqslant q$ ). Let $S$ be the subring with 1 of $R^{\prime \prime}$ generated by the elements $s_{1}, \ldots, s_{q}, s_{q+1}, \ldots, s_{r}, f_{1}, \ldots, f_{q}$ and their inverses.

In the event that $R$ is a domain, let $R^{\prime \prime}$ be its field of fractions and $s_{1}, \ldots, s_{q}, s_{q+1}, \ldots, s_{r}$ be the collection of all nonzero entries appearing in the generating matrices $g_{1}, \ldots, g_{m}$ of $G$. Again we may assume that $s_{1}, \ldots, s_{q}$ are of infinite order and that $s_{q+1}, \ldots, s_{r}$ are respectively of finite order $e_{q+1}, \ldots, e_{r}$. Put $f_{i}=1+s_{i}(1 \leqslant i \leqslant q)$ and then let $S$ be the subring with 1 of $R^{\prime \prime}$ generated by the elements $s_{1}, \ldots, s_{q}, s_{q+1}, \ldots, s_{r}, f_{1}, \ldots, f_{q}$ and their inverses.

5. We need a special subgroup of $\operatorname{Tr}(n, S)$, viz,

$$
G(n, S)=\left\{\left(a_{i, j}\right) \in \operatorname{Tr}(n, S) \mid a_{i, i} \in S^{*}(1 \leqslant i \leqslant n)\right\}
$$

where $S^{*}$ is the multiplicative subgroup of $S$ generated by the elements $s_{1}, \ldots, s_{q}, s_{q+1}, \ldots, s_{r}, f_{1}, \ldots, f_{q}$. By the construction of $S$ we see that $G$ is embedded in $G(n, S)$. 
Notice that $G(1, S)$ is isomorphic to $S^{*}$. Let $n \geqslant 2$. If $A$ is an element of $G(n, S)$ then we can write

$$
A=\left(\begin{array}{cccc}
a_{1} & 0 & \cdots & 0 \\
a_{2} & & & \\
\vdots & & A^{\prime} & \\
a_{n} & &
\end{array}\right)
$$

where $A^{\prime} \in G(n-1, S)$ is simply the lower-right $(n-1) \times(n-1)$ submatrix of $A$. By straightforward multiplication of the lower triangular matrices we see that the mapping $\theta$ given by $\theta: A \mapsto A^{\prime}$ defines a homomorphism of $G(n, S)$ onto $G(n-1, S)$ with kernel

$$
K(n, S)=\left\{A \in G(n, S) \mid A=\left(\begin{array}{cccc}
a_{1} & 0 & \cdots & 0 \\
a_{2} & & \\
\vdots & 1_{n-1} &
\end{array}\right)\right\}
$$

Thus for $n \geqslant 2$ we have a short exact sequence of groups

$$
1 \rightarrow K(n, S) \rightarrow G(n, S) \rightarrow G(n-1, S) \rightarrow 1
$$

with $G(1, S)$ isomorphic to $S^{*}$.

6. We shall need, in the sequel, an analysis of $K(n, S)$. Let $n \geqslant 2$. Then for each $i(2 \leqslant i \leqslant n)$ and $a \in S$ let $A_{i}(a)$ be the $n \times n$ lower triangular matrix with entry $a$ in the $i$ th row and first column, 1 everyplace on the main diagonal, zero elsewhere. For each $b \in S^{*}$ let $D(b)$ denote the $n \times n$ diagonal matrix with entry $b$ in the first position on the main diagonal and the entry 1 thereafter. Obviously these matrices are contained in $K(n, S)$. We list some of the easily verified properties of these matrices:

$$
\begin{array}{lr}
D\left(b_{1}\right)^{n_{1}} \cdots D\left(b_{r}\right)^{n_{r}}=D\left(b_{1}^{n_{1}} \cdots b_{r}^{n_{r}}\right) & \left(n_{1}, \ldots, n_{r} \in Z\right), \\
{\left[D(b), D\left(b^{\prime}\right)\right]=1_{n},} & \\
A_{i}\left(a_{1}\right)^{n_{1}} \cdots A_{i}\left(a_{r}\right)^{n_{r}} & \\
\quad=A_{i}\left(n_{1} a_{1}+\cdots+n_{r} a_{r}\right) & \left(n_{1}, \ldots, n_{r} \in Z, 2 \leqslant i \leqslant n\right), \\
{\left[A_{i}(a), A_{i^{\prime}}\left(a^{\prime}\right)\right]=1_{n}} & \left(2 \leqslant i, i^{\prime} \leqslant n\right), \\
A_{i}(a)^{D(b)}=A_{i}(a b) & (2 \leqslant i \leqslant n),
\end{array}
$$

and 


$$
D(b) A_{2}\left(a_{2}\right) \cdots A_{n}\left(a_{n}\right)=\left(\begin{array}{cccc}
b & 0 & \cdots & 0 \\
a_{2} & & \\
\vdots & 1_{n-1} & \\
a_{n} & &
\end{array}\right) .
$$

If we put $A_{i}=A_{i}(1)$ for each $i(2 \leqslant i \leqslant n)$, it follows that we have proved that

$$
K(n, S)=\operatorname{gp}\left(A_{2}, \ldots, A_{n}, D\left(s_{i}\right), D\left(f_{j}\right) \mid 1 \leqslant i \leqslant r, 1 \leqslant j \leqslant q\right)
$$

is a finitely generated metabelian group.

7. We are now ready to prove that $G(n, S)$ is finitely presented. Recall from $\S 5$ that $G(1, S)$ is isomorphic to $S^{*}$ and for each $n \geqslant 2$ we have a short exact sequence of groups

$$
1 \rightarrow K(n, S) \rightarrow G(n, S) \rightarrow G(n-1, S) \rightarrow 1 .
$$

It is clear that $G(1, S)$ is finitely presented. Inductively, for $n \geqslant 2$, we shall assume that $G(n-1, S)$ is finitely presented. Then, in view of the displayed short exact sequence of groups above, to show $G(n, S)$ is finitely presented it will be sufficient to show that $K(n, S)$ is finitely presented for each $n \geqslant 2$.

Let $n \geqslant 2$ be fixed. To show $K(n, S)$ is finitely presented we shall construct a finitely presented metabelian group $M$ which has $K(n, S)$ as a homomorphic image. Since the class of finitely presented metabelian groups is an image closed class (Hall [11]), this will insure that $K(n, S)$ is finitely presented.

We shall present $M$ on the generators

$$
X=\left\{a_{2}, \ldots, a_{n}, t_{1}, \ldots, t_{q}, t_{q+1}, \ldots, t_{r}, u_{1}, \ldots, u_{q}\right\}
$$

where the integers $n, q, r$ are the integers occurring in the description of $K(n, S)($ see $(3))$.

The defining relations of $M$ are of four kinds. First we have the power relations

$$
t_{i}^{e_{i}}=1 \quad(q+1 \leqslant i \leqslant r)
$$

where here the positive integer $e_{i}$ is the order of the element $s_{i}(q+1 \leqslant i$ $\leqslant r$ ). Next we have the commutativity relations

$$
\begin{array}{ll}
{\left[t_{i}, t_{j}\right]=1} & (1 \leqslant i, j \leqslant r), \\
{\left[t_{i}, u_{j}\right]=1} & (1 \leqslant i \leqslant r, 1 \leqslant j \leqslant q), \\
{\left[u_{i}, u_{j}\right]=1} & (1 \leqslant i, j \leqslant q) .
\end{array}
$$


Thirdly we have the commutativity relations for the conjugates of the generators $a_{i}$ :

$$
\begin{aligned}
& {\left[a_{i}, a_{j}^{w}\right]=1 \text { where } 2 \leqslant i, j \leqslant n, \text { and }} \\
& w \in\left\{t_{1}^{n_{1}} \cdots t_{q}^{n_{q}} t_{q+1}^{n_{q+1}} \cdots t_{r}^{n_{r}} \mid-d_{i} \leqslant n_{i} \leqslant d_{i}(1 \leqslant i \leqslant q),\right. \\
& \left.0 \leqslant n_{i}<e_{i}(q+1 \leqslant i \leqslant r)\right\} .
\end{aligned}
$$

Here the positive integer $d_{i}$ occurring in (7) is simply the "degree" of the element $f_{i}$ (see $\left.(1)\right)(1 \leqslant i \leqslant q)$. Finally we have the defining relations giving the action of the elements $u_{j}$ on the elements $a_{i}$ :

$$
a_{i}^{u_{j}}=a_{i}^{f_{j}\left(t_{j}\right)} \quad(2 \leqslant i \leqslant n, 1 \leqslant j \leqslant q),
$$

where $f_{j}(x)=1+c_{j, 1} x+\cdots+c_{j, d_{j}-1} x^{d_{j}-1}+x^{d_{j}}$ is an element of the polynomial ring $Z[x]$ (again see (1)).

We emphasize that $M$ is the group generated by the set $X$ given by (4) subject to the defining relators (5), (6), (7) and (8). $M$ is patently finitely presented. Moreover, upon appealing to Lemma $2, M$ is also metabelian.

Now define a map $\theta$ from $M$ to $K(n, S)$ by the rule

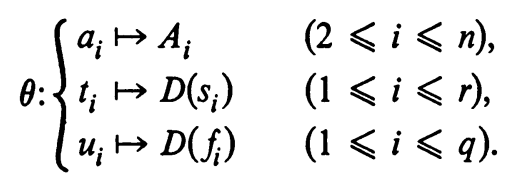

It follows, upon examining (1), the relations satisfied in $K(n, S)$ (see (2)) and the defining relations (5)-(8) of $M$, that $\theta$ extends to a homomorphism from $M$ onto $K(n, S)$. Hence $K(n, S)$ is finitely presented as desired.

Thus we have proved Theorem 1.

It is worth noting that the group $G(n, S)$ of this theorem actually has a normal series of subgroups

$$
1=H_{0} \leqslant H_{1} \leqslant \cdots \leqslant H_{n}=G(n, S)
$$

in which the sucessive factor groups $H_{i+1} / H_{i}(i=0, \ldots, n-1)$, are all finitely presented and metabelian. In particular $G(n, S)$ satisfies the maximal condition for normal subgroups.

8. It is now easy to prove

THFOREM 2. Every finitely generated solvable linear group can be embedded in a finitely presented solvable linear group.

Proof. Let $G$ be a finitely generated solvable subgroup of $\mathrm{GL}(n, k)$. We can suppose that $k$ is algebraically closed. Hence there exists a subgroup $H$ of $G$ 
with finite index and an element $x$ of $\operatorname{GL}(n, k)$ such that $H^{x}$ is a subgroup of $\operatorname{Tr}(n, k)$ (A. I. Mal'cev [13]). Replacing $G$ by $G^{x}$ if necessary, we can assume that $H$ is a subgroup of $\operatorname{Tr}(n, k)$. Since $G$ is finitely generated and $H$ has finite index in $G, H$ is also finitely generated and we can even assume that $H$ is a normal subgroup of $G$. Thus $H$ is a subgroup of a finitely presented subgroup $K$ of $\operatorname{Tr}(n, k)$. Since $G / H$ is finite, $G$ can be embedded in the standard (restricted) wreath product $H \sim G / H$ of $H$ by $G / H$ (see Krasner and Kaloujnine [12]). Let $W=K \sim G / H$ be the standard wreath product of $K$ by $G / H$ and notice that $H \sim G / H$ is a subgroup of $W$. Therefore $G$ has been embedded in $W$.

We observe that since $K$ is finitely presented and $G / H$ is finite it follows that $W$ is finitely presented. Next, since $W$ is the wreath product of solvable groups, it too is solvable. Finally, $K$ is a subgroup of $\operatorname{GL}(n, k)$ and $G / H$ is finite so that $W$ is isomorphic to a linear group over $k$ (see B. A. F. Wehrfritz [17, p. 151]).

9. In this section we shall prove Corollary 1 by providing an embedding of a countable free nilpotent group of any given class $c$ into a finitely generated solvable linear group. The proofs of Corollary 2 and Corollary 3 follow immediately upon examining their linear representations provided in [8] and [9] respectively.

To begin note that every countable free nilpotent group of any given class $c$ has a faithful representation in $\operatorname{Tr}(n, k)$ for some positive integer $n$ and some suitably chosen commutative field $k$. For example, let $x$ be a single indeterminate over $Z$ and for each $r=1,2, \ldots$ let $x_{r}=\left(x_{r}(i, j)\right)$ be an element of $\operatorname{Tr}(c+1, k)$ where $k$ is the quotient field of the polynomial ring $Z[x]$ and

$$
x_{r}(i, j)= \begin{cases}0 & (i-j \geqslant 2 \text { or } i-j \leqslant-1), \\ 1 & (i-j=0), \\ x^{2^{\text {cri-2 }}} & (i-j=1)\end{cases}
$$

(here $i$ denotes the row and $j$ the column of the entry $\left.x_{r}(i, j)\right)$. Then $F=\operatorname{gp}\left(x_{r} \mid r=1,2, \ldots\right)$ is a free nilpotent group of class $c$ on the generating set $X=\left\{x_{r} \mid r=1,2, \ldots\right\}$ (see again B. A. F. Wehrfritz [17, p. 34]). It is easy to embed $F$ in a finitely generated subgroup $G$ of $\operatorname{Tr}(c+1, k)$. In fact let $A_{i+1, i}$ be the matrix in $\operatorname{Tr}(c+1, k)$ with entry 1 in the $(i+1)$ th row and $i$ th column, 1 everyplace on the main diagonal, zero row and $i$ th column, 1 everyplace on the main diagonal, zero elsewhere $(1 \leqslant i \leqslant c)$. For each $j(1 \leqslant j \leqslant c)$ let $D_{j}$ be the diagonal matrix in $\operatorname{Tr}(c+1, k)$ with entry $x$ in the jth position on the main diagonal and 1 elsewhere. Let $G$ be the subgroup of $\operatorname{Tr}(c+1, k)$ generated by the elements $A_{i+1, i}(1 \leqslant i \leqslant c), D_{j}(1 \leqslant j \leqslant c)$. An easy calculation shows that for each $r=1,2, \ldots$ 


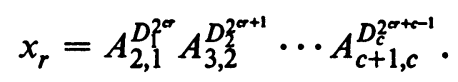

Thus $F$ is a subgroup of $G$. It follows that we have proved Corollary 1 .

10. Finally we remark that V. N. Remeslennikov's example [14] of a finitely presented solvable group $G$ without the maximal condition for normal subgroups is incorrect. In view of the importance of the problem involved we shall indicate how Remeslennikov's example fails. To do this we first recall that

$$
G=\left\langle a, x, y ;[x, y], a^{y}=a a^{x},\left[a, a^{x}\right]^{x}=\left[a, a^{x}\right],\left[\left[a, a^{x}\right], a\right]\right\rangle .
$$

It turns out that $G^{\prime}=\mathrm{gp}_{G}(a)$, the normal closure of $a$ in $G$, is nilpotent of class 2 and that $G$ is a split extension of $G^{\prime}$ by $B=\operatorname{gp}(x, y)$ a free abelian group on $x$ and $y$.

We let $K=\mathrm{gp}_{G}\left(\left[a, a^{x}\right]\right)$ be the normal subgroup of $G$ generated by the element $\left[a, a^{x}\right]$. The factor group $G / K$ can be presented by $(\bar{w}=w K, w$ in $G)$,

$$
G / K=\left\langle\bar{a}, \bar{x}, \bar{y} ;[\bar{x}, \bar{y}], \bar{a}^{\bar{y}}=\bar{a} \bar{a}^{\bar{x}},\left[\bar{a}, \bar{a}^{\bar{x}}\right]\right\rangle .
$$

It follows from Lemma 2 that $G / K$ is metabelian. Since $G / K$ is finitely generated it satisfies the maximal condition for normal subgroups (Hall [11]). Therefore $G$ satisfies the maximal condition for normal subgroups if and only if $K$ satisfies the maximal condition for its $G$-invariant subgroups (a subgroup of $K$ is $G$-invariant if it is a normal subgroup of $G$ ).

Let $L=\operatorname{gp}\left(\left[a^{x^{i} y^{j}}, a^{x^{i+1} y^{j}}\right] \mid i, j\right.$ integers $)$. Then $L$ is a subgroup of the center of $G^{\prime}$. Since every element of $G$ has the form $x^{m} y^{n} g^{\prime}$ where $m, n$ are integers and $g^{\prime}$ is an element of $G^{\prime}$, it follows that $L$ is a normal subgroup of $G$. Because $L$ contains the element $\left[a, a^{x}\right], K=L$. We let $R=Z B$ be the integral group ring of $B$. Then $R$ is noetherian. $K$ becomes a right $R$-module in the usual way (the action is conjugation by elements of $B$ extended to $R$ in the natural way) and every normal subgroup of $G$ contained in $K$ is a right $R$ submodule of the right $R$-module $K$. But clearly $K$ is finitely generated as a right $R$-module, indeed by $\left[a, a^{x}\right]$. Since $R$ is noetherian, $K$ is also noetherian. Hence $K$ satisfies the maximal condition for its $G$-invariant subgroups and therefore $G$ satisfies the maximal condition for normal subgroups.

\section{REFERENCES}

1. M. F. Atiyah and I. G. Macdonald, Introduction to commutative algebra, Addison-Wesley, Reading, Mass., 1969. MR 39 \#4129.

2. Gilbert Baumslag, Subgroups of finitely presented metabelian groups, J. Austral. Math. Soc. 16 (1973), 98-110. MR 48 \#11324.

3. 125-127. MR 48 \#6252. 
4. - A finitely presented metabelian group with a free abelian derived group of infinite rank, Proc. Amer. Math. Soc. 35 (1972), 61-62. MR 45 \#8710.

5. - Finitely presented metabelian groups, Proc. Second Internat. Conf. on Theory of Groups (Canberra, 1973), Lecture Notes in Math., vol. 372, Springer-Verlag, Berlin and New York, 1974, pp. 65-74. MR 49 \#9054.

6. - Multiplicators and metabelian groups, J. Austral. Math. Soc. (to appear).

7. - On the subalgebras of certain finitely presented algebras, Bull. Amer. Math. Soc. 82 (1976), 95-98.

8. D. I. Ėìdel'kind, Exact representations for free groups, Algebra i Logika 10 (1971), 449-473 = Algebra and Logic 10 (1971), 281-296 (1973). MR 46 \#9183.

9. C. K. Gupta, A faithful matrix representation for certain centre-by-metabelian groups, J. Austral. Math. Soc. 10 (1969), 451-464. MR 41 \#5513.

10. C. K. Gupta and N. D. Gupta, On the linearity of free nilpotent-by-abelian groups, J. Algebra 24 (1973), 293-302. MR 46 \#9164.

11. Philip Hall, Finiteness conditions for soluble groups, Proc. London Math. Soc. (3) 4 (1954), 419-436. MR 17, 344.

12. L. Kaloujnine and Marc Krasner, Produit complete des groupes de permutations et le problème d'extension des groupes. III, Acta Sci.Math. (Szeged) 14 (1951), 69-82.

13. A. I. Mal'cev, On certain classes of infinite solvable groups, Mat. Sb. 28 (70) (1951), 567-588; English transl., Amer. Math. Soc. Transl. (2) 2 (1956), 1-21. MR 13, 203.

14. V. N. Remeslennikov, An example of a finitely defined solvable group without the maximality condition for normal subgroups, Mat. Zametki 12 (1972), 287-293 = Math. Notes 12 (1972), 606-609 (1973). MR 47 \#8694.

15. - Finite approximability of metabelian groups, Algebra i Logika 7 (1968), 106-113= Algebra and Logic 7 (1968), 268-272.

16. N. S. Romanovskiï, Bases for identical relations for certain matrix groups, Algebra i Logika 10 (1971), 401-406 = Algebra and Logic 10 (1971), 248-251. MR 46 \#3640.

17. B. A. F. Wehrfritz, Infinite linear groups, Ergebnisse Math. Grenzgebiete, Band 76, Springer-Verlag, Berlin and New York, 1973. MR 49 \#436.

Department of Mathematics, Graduate School and University Center (CUNY), New YORK, NEW YORK 10036

Current address: Department of Mathematics, University of Manitoba, Winnipeg, Manitoba, Canada R3T 2N2 